\title{
Long Memory Volatility Models in R: Application to a Regional Blue Chips Index
}

\author{
Visar MALAJ (PhD) \\ 1 University of Tirana, Faculty of Economy, Department of Economics \\ visarmalaj@feut.edu.al
}

Arben MALAJ (Prof. Asoc.)

2University of Tirana, Faculty of Economy, Department of Economics

arbenmalaj@feut.edu.al

\begin{abstract}
An increasing number of researchers and analysts have formulated in the last decade innovative statistical equations for modelling and forecasting the volatility of financial returns. The evolution of technology and new software development have contributed positively to emerging economies, as well as in the improvement of market competition. We investigate in this study some experimental extensions of the multiplicative error model, which has been introduced by Engle (2002) for positive valued processes and it is specified as the product of a conditionally autoregressive scale factor and an innovation process with positive support. We use R 3.1.3 for the optimization, a modern software for statistical computing and graphics. Empirical analysis is carried out starting from May 2009 to January 2015. The equations fit fairly well the volatility of 'STOXX Balkan 50 Equal Weight' Index, which represents leading blue chips from eight Balkan countries in terms of free-float market cap. The models seem to absorb completely the slow decay of the global autocorrelation function.
\end{abstract}

Keywords: Stock Market Data, Volatility, MEM models, Balkan stock index.

\section{Introduction}

The volatility process is associated with the conditional variance of financial returns over time. It is a subject to be taken into consideration while assessing the risk of an investment in financial assets. A relatively high level of volatility means that the corresponding asset price tends to large variations over time, therefore the investor can record high profits or high losses. The realized volatility is a positive valued process with a highly persistent temporal dependency, indicated by a slow hyperbolic decay of the autocorrelation function. Granger and Joyeux (1980) and Hosking (1981) suggest the use of fractionally integrated autoregressive moving average (ARFIMA) model in order to capture the long term persistence in time series of many fields. Engle and Russell (1998) propose a different method for the analysis of data which arrive at irregular interval such as financial transaction series. The authors define a new statistical model called autoregressive conditional duration (ACD), which can be considered part of the generalized autoregressive conditional heteroskedasticity (GARCH) model, introduced by Bollerslev (1986). Engle (2002) extends the research suggesting the use of the multiplicative error model (MEM) for modelling non-negative processes. This model specifies an error that is multiplied times the conditional expectation that follows a GARCH dynamic. Brownlees, Cipollini and Gallo (2011) focus on the different possible specifications of the persistence in the conditional mean using applications on the volatility of components of the S\&P100 index, following the logic of Engle and Lee (1999). Cipollini, Engle and Gallo (2013) suggest an extension to the vector MEM model defined by Cipollini, Engle and Gallo (2006), where the conditional expectation is given as a function of its past and the conditional expectations of other variables. The authors follow a semiparametric method lacking the necessity of specifying the probabilistic distribution function or the particular copula function. Barigozzi, Brownlees, Gallo and Veredas (2014) expand the MEM model introduced by Engle (2002), decomposing the conditional expectation as the product of a systematic trend and an idiosyncratic dynamic component. This model, named SeminonParametric Vector MEM (SPVMEM), can be used to estimate panels of market activity, risk and liquidity measures.

We suggest in this paper an interesting flexible MEM specification in which the process for realized volatility can be seen as a mixture of two Gamma MEMs with different coefficients for the conditional expectation and different shape parameters for the Gamma (De Luca and Gallo, 2007; Gallo and Otranto, 2014). We extend this framework even further adopting a mixed Gamma model for a MEM with fixed parameters which depend on the value of the past (Lanne, 2006; Ahoniemi and Lanne, 2013). 
The paper is organized as follows. Section 2 establishes the notation by summarizing some of the features of the multiplicative error model. Section 3 contains features of the equations and some empirical results illustrating the behaviour of the two MEM specifications for the realized volatility of 'STOXX Balkan 50 Equal Weight' Index. Section 4 concludes.

\section{Theoretical framework}

Let us consider $r_{t}$ to be the daily log return at time $t$ with negligible mean. We use a common indicator of volatility $y_{t}$, the daily squared return. The general structure of a multiplicative error model was outlined by Engle (2002) for realized volatility. A linear $\operatorname{MEM}(p, q)$ is given by:

$$
y_{t}=\mu_{t} \varepsilon_{t}
$$

where, conditionally to information $I_{t-1}: \mu_{t}$ is a non-negative predictable process. The $\mu_{t}$ evolution depends on a vector of unknown parameters $\theta, \mu_{t}=\mu_{t}(\vartheta)$. $\varepsilon_{t}$ follows a distribution with nonnegative support, with mean 1 and unknown variance $\sigma^{2}$. We have:

$$
\varepsilon_{t} \mid I_{t-1} \sim D\left(1, \sigma^{2}\right) \text {. }
$$

These definitions provide us:

$$
\begin{aligned}
& E\left(y_{t} \mid I_{t-1}\right)=\mu_{t} \\
& V\left(y_{t} \mid I_{t-1}\right)=\sigma^{2} \mu_{t}^{2}
\end{aligned}
$$

In order to close the model, we adopt a density function for $\varepsilon_{t}$ and specify an equation for the conditional mean $\mu_{t}$. A common choice in many studies, is to use the exponential distribution, a particular case of the Gamma distribution. We have:

$$
\varepsilon_{t} I_{t-1} \sim \operatorname{Gamma}(\phi, 1 / \phi),
$$

where $\phi>0, E\left(\varepsilon_{t} \mid I_{t-1}\right)=1$ and $V\left(\varepsilon_{t} \mid I_{t-1}\right)=1 / \phi$. The density function conditioned to the innovation $\varepsilon_{t}$ is:

$$
f\left(\varepsilon_{t} \mid I_{t-1}\right)=\frac{1}{\Gamma(\phi)} \phi^{\phi} \varepsilon_{t}^{\phi-1} \exp \left\{-\phi \varepsilon_{t}\right\} \text {. }
$$

We can conclude that $y_{t}$ follows a Gamma conditioned distribution

$$
y_{t} \mid I_{t-1} \sim \operatorname{Gamma}\left(\phi, \mu_{t} / \phi\right)
$$

with density function:

$$
f\left(y_{t} \mid I_{t-1}\right)=\frac{1}{\Gamma(\phi)} \frac{\phi^{\phi}}{\mu_{t}^{\phi}} y_{t}^{\phi-1} \exp \left\{-\phi \cdot y_{t} / \mu_{t}\right\} \text {. }
$$

So, we have in this case:

$$
\begin{aligned}
& E\left(y_{t} \mid I_{t-1}\right)=\phi \cdot \frac{\mu_{t}}{\phi}=\mu_{t} \\
& V\left(y_{t} \mid I_{t-1}\right)=\phi \cdot \frac{\mu_{t}^{2}}{\phi^{2}}=\frac{\mu_{t}^{2}}{\phi} .
\end{aligned}
$$

With regards to $\mu_{t}$, we consider the simplest specification, the $\operatorname{GARCH}(\mathrm{p}, \mathrm{q})$. 


$$
\mu_{t}=\omega+\sum_{i=1}^{p} \alpha \cdot y_{t-i}+\sum_{j=1}^{q} \beta_{j} \cdot \mu_{t-j} .
$$

We estimate the parameters included in $\mu_{t}$, let's call them $\theta$. The log-likelihood function useful for the estimation is:

$$
\log L\left(y_{t} ; \theta\right)=l\left(y_{t} ; \theta\right)=c-\phi \sum_{t=1}^{T}\left(\log \mu_{t}+\frac{y_{t}}{\mu_{t}}\right),
$$

where $\mathrm{c}$ is an irrelevant constant to the estimation method depending on $\Phi$. We maximize the function with respect to $\theta$ :

$$
\sum_{t=1}^{T}\left(\frac{x_{t}-\mu_{t}}{\mu_{t}^{2}}\right) \frac{\partial \mu_{t}}{\partial \theta}=0 \text {. }
$$

We obtain the our estimators $\hat{\theta}_{S M V}$ using iterative procedures. We have:

$\hat{\theta}_{S M V}=\operatorname{argmax} \max _{\theta} l\left(y_{t} ; \theta\right)$

The asymptotic distribution of the estimators under regularity conditions will be:

$$
\begin{aligned}
& \sqrt{T}\left(\hat{\theta}_{n}-\theta\right)^{a s y} \sim N\left(0, V_{a s}\right) \\
& \hat{\theta}_{n}^{a s y} \sim N\left(\theta, \frac{1}{T} V_{a s}\right),
\end{aligned}
$$

where $V_{a s}=J^{-1}$ is the asymptotic variance. We have:

$$
\begin{aligned}
& J=E\left[-\frac{\partial^{2} l\left(y_{t} ; \theta\right)}{\partial \theta \partial \theta^{\prime}}\right], \\
& \hat{J}=-\frac{1}{T} \sum_{i=1}^{T} \frac{\partial^{2} l\left(y_{t} ; \theta\right)}{\partial \theta \partial \theta^{\prime}}=\left.\frac{1}{T} \sum_{i=1}^{T} \frac{\partial l\left(y_{t} ; \theta\right)}{\partial \theta} \frac{\partial l\left(y_{t} ; \theta\right)}{\partial \theta^{\prime}}\right|_{\theta=\theta^{\prime}}
\end{aligned}
$$

If order to verify the null hypothesis $H_{0}: \theta_{i}=\theta_{i, 0}$, we use the Student's t test:

$t=\frac{\hat{\theta}_{i}-\theta_{i, 0}}{\sqrt{V_{i i}}}$

where $\hat{\theta}_{i}$ is the $i$-th row vector and $V_{i i}$ is the (i,i) element of the $\frac{1}{T} \hat{V}_{a s}=\frac{1}{T} \hat{J}^{-1}$ matrix.

\section{MEM equations and application}

Initially, we consider an interesting formulation of MEM models (Lanne, 2006; Ahoniemi and Lanne, 2013), where the innovation term $\varepsilon_{t}$ follows a mixed gamma distribution with constant parameters. The innovation mean is unitary respecting the establishment of the previous section. Let's call $y_{t}$ the realized volatility of a generic stock. We have:

$y_{t}=\mu_{t} \varepsilon_{t}$.

The innovation is defined as follows: 
$\varepsilon_{t}=\left\{\begin{array}{l}\varepsilon_{1, t} \text { with prob. } \pi \\ \varepsilon_{2, t} \text { with prob. }(1-\pi)\end{array}\right.$,

where $0<\pi<1$ and

$$
\begin{aligned}
& \varepsilon_{1, t} \sim \operatorname{Gamma}\left(\gamma_{1}, 1 / \gamma_{1}\right) \\
& \varepsilon_{2, t} \sim \operatorname{Gamma}\left(\gamma_{2}, 1 / \gamma_{2}\right) .
\end{aligned}
$$

The general form of the conditional mean equation is:

$$
\begin{aligned}
& \mu_{1 t}=\omega_{1}+\sum_{i=1}^{q} \alpha_{1 i} \cdot y_{t-i}+\sum_{i=1}^{p} \beta_{1 j} \cdot \mu_{1, t-j} \\
& \mu_{2 t}=\omega_{2}+\sum_{i=1}^{q} \alpha_{2 i} \cdot y_{t-i}+\sum_{i=1}^{p} \beta_{2 j} \cdot \mu_{2, t-j} .
\end{aligned}
$$

We consider also a model, belonging to the MEM family, with an innovation term distributed according to a mixture of Gamma with variable weights (De Luca and Gallo , 2007; Gallo and Otranto, 2014). The overall mean of the innovation term is always unitary. So, we have:

$$
\begin{aligned}
& \varepsilon_{t}=\left\{\begin{array}{l}
\varepsilon_{1, t} \text { with prob. } \quad \pi_{t} \\
\varepsilon_{2, t} \text { with prob. }\left(1-\pi_{t}\right)
\end{array}\right. \\
& \varepsilon_{1, t} \sim \operatorname{Gamma}\left(\lambda_{1}, \gamma_{1}\right) \\
& \varepsilon_{2, t} \sim \operatorname{Gamma}\left(\lambda_{2}, \gamma_{2, t}\right)
\end{aligned}
$$

where $0<\pi<1$. We suppose that weights depend on their past and on the previous value of realized volatility:

$$
\pi_{t}=\frac{\exp \left\{\delta_{0}+\delta_{1} \cdot y_{t-1}+\delta_{2} \pi_{t-1}\right\}}{1+\exp \left\{\delta_{0}+\delta_{1} \cdot y_{t-1}+\delta_{2} \pi_{t-1}\right\}}
$$

Imposing the unitary mean constraint, we have:

$$
\gamma_{2, t}=\frac{1-\pi_{t} \lambda_{1} \gamma_{1}}{\left(1-\pi_{t}\right) \lambda_{2}} \text {. }
$$

We call the two models respectively, LANNE and DLG in our analysis. After defining the regression models, it is important to confirm the goodness of fit of the model and the statistical significance of the estimated parameters. In simple linear models the errors follow a normal distribution, but in some regressions based on mixture of distributions this hypothesis may not be valid. The method used in this paper is based on the so-called probability integral transformation of Rosenblatt (1952), which is a universally approach of transforming a set of dependent variables into independent uniform distributed variables. Dunn and Smyth (1996) define and apply the quantile residuals to regression models with independent responses.

Let $F(y ; \mu, \phi)$ be the cumulative distribution function of $\mathrm{P}(\mu, \phi)$. If $F$ is continuous, then the $F\left(y_{i} ; \mu, \phi\right)$ are uniformly distributed on the unit interval. The quantile residuals are defined by

$$
r_{q, i}=\Phi^{-1}\left\{F\left(y_{i} ; \hat{\left.\mu_{i}, \hat{\phi}\right)}\right\}\right.
$$

where $\Phi()$ is the cumulative distribution function of the standard normal. Quantile residuals are independent and follow a standard normal distribution if the model is correctly specified and the parameters are consistently estimated. 
We start with considering the realized volatility of 'STOXX Balkan 50 Equal Weight' Index, which represents the largest and most liquid companies across eight Balkan countries. The index covers 50 stocks from Bulgaria, Croatia, Greece, the Former Yugoslav Republic of Macedonia (FYROM), Romania, Serbia, Slovenia and Turkey. We consider the period from $1^{\text {st }}$ May 2009 to $30^{\text {th }}$ January 2015. The time series of daily realized volatility has been built as the square root of realized variance. Figures 1 displays the plot of realized volatility. Figure 2 shows the estimated global autocorrelation function and partial autocorrelation function of realized volatility which exhibit a very slow decay, that is a long-memory pattern.

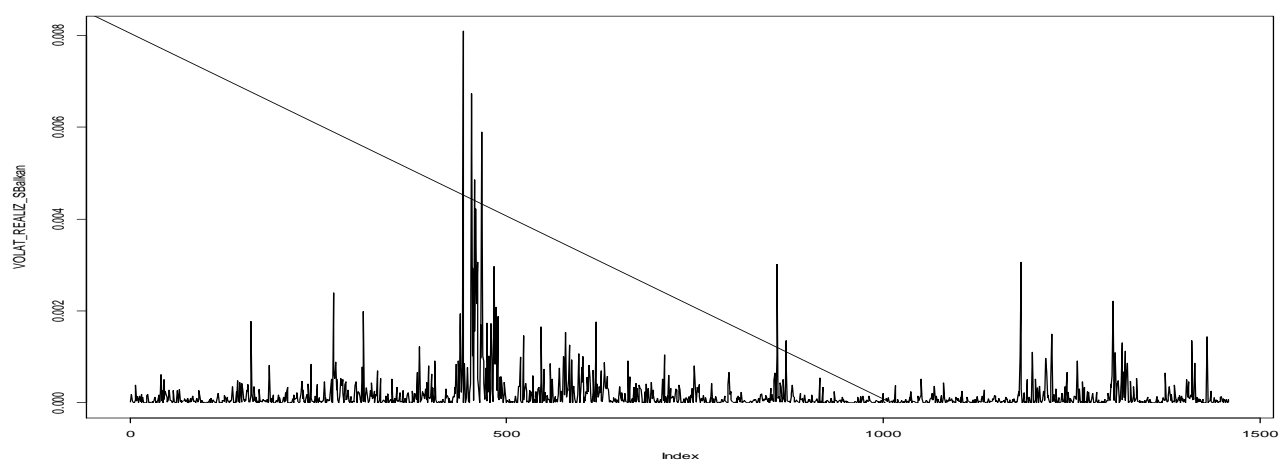

Figure 1. Time series of 'STOXX Balkan 50 Equal Weight' realized volatility.

Series VOLAT_REALIZ_SBalkan

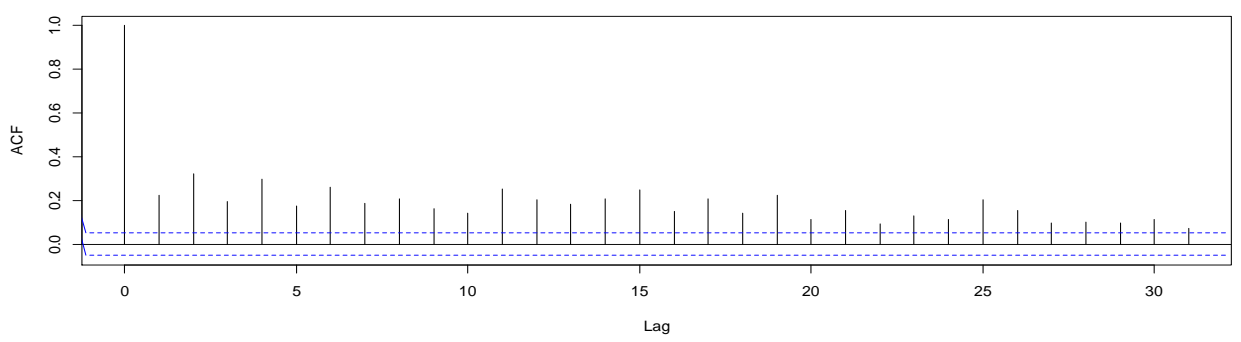

Series VOLAT_REALIZ_SBalkan

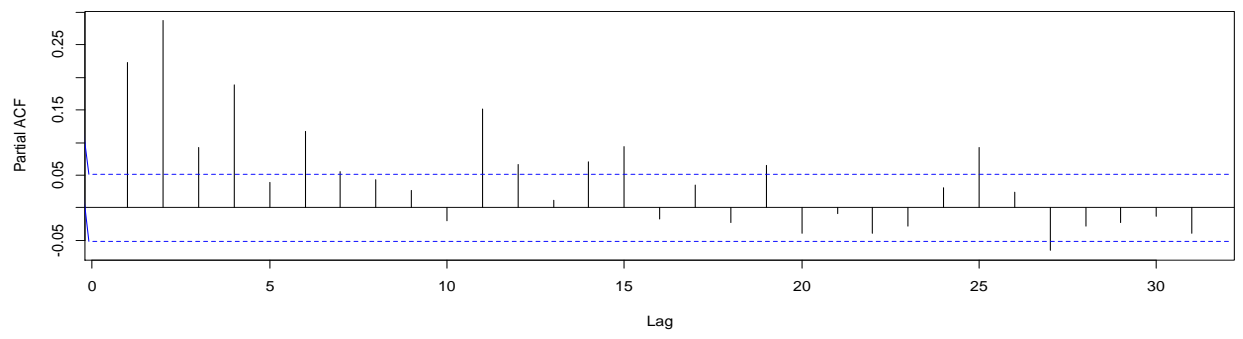

Figure 2. Global Autocorrelation Function and Partial Autocorrelation Function for 'STOXX Balkan 50 Equal Weight' realized volatility.

We try to model the realized volatility series with both possible specifications of MEM models. Let's see what happens with the LANNE specification. This is the conditional mean equation, where $p=2$ and $q=1$ : 


$$
\mu_{t}=\omega+\alpha_{1} \cdot y_{t-1}+\alpha_{2} \cdot y_{t-2}+\beta_{1} \mu_{t-1} \text {. }
$$

We can see in the tables below the estimate, the standard error, the statistic test value and the observed $p$-value for each of the seven parameters. We simply observe the last column of the table where is calculated the $p$-value for the statistical test. We can fix a priori a p-value of $5 \%$ for acceptance of the null hypothesis. So, we accept ' $\mathrm{HO}$ : The parameter is null' if the observed $p$-value is greater than $5 \%$ and vice versa if it is less than $5 \%$. As we can see from the table we refuse the null hypothesis in all cases, a positive indicator for the model evaluation.

\begin{tabular}{|l|l|l|}
\hline Parameter & Estimation & p-value \\
\hline$\pi$ & 0.067 & $\sim 0$ \\
\hline$\gamma_{1}$ & 5.634 & $\sim 0$ \\
\hline$\omega_{1}$ & 0.127 & $\sim 0$ \\
\hline$\alpha_{11}$ & 0.374 & $\sim 0$ \\
\hline$\beta_{11}$ & 0.749 & $\sim 0$ \\
\hline$\gamma_{2}$ & 11.564 & $\sim 0$ \\
\hline$\omega_{2}$ & 0.011 & $\sim 0$ \\
\hline$\alpha_{21}$ & 0.378 & $\sim 0$ \\
\hline$\beta_{21}$ & 0.769 & $\sim 0$ \\
\hline & & \\
\hline
\end{tabular}

\section{Table 1. Parameters test, LANNE model.}

Let's check now if the model is specified correctly or not using the quantile residuals method mentioned above. We have represented in figure 3 (from the left to the right) the quantile residual graph, the quantile-quantile graph (if the distribution approximates the normal standard one), the global autocorrelation (ACF) and the partial autocorrelation (PACF) of the quantile residuals and of the squared quantile residuals for LANNE model. We observe that the approximation is generally good. 

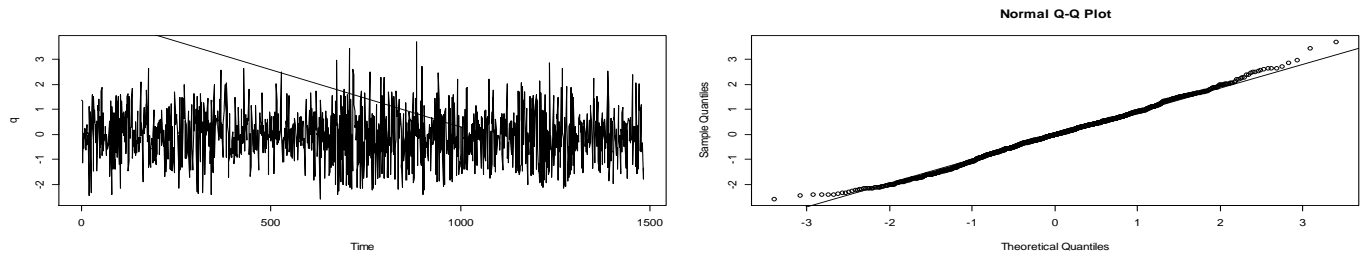

Series q

Series q
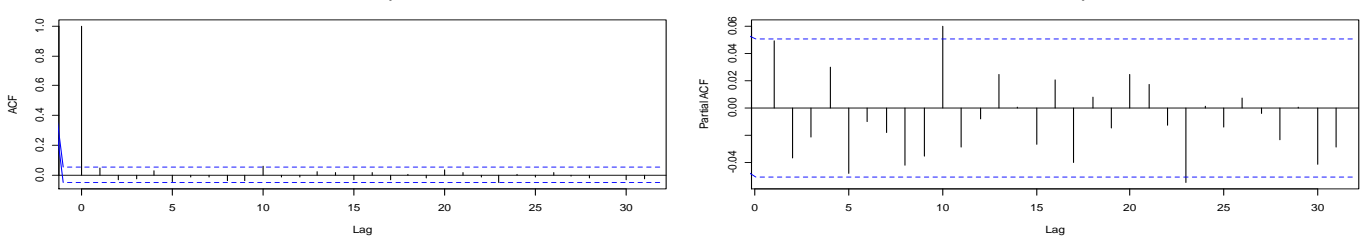

Series $q^{\wedge} 2$

Series $\mathbf{q}^{\wedge 2}$
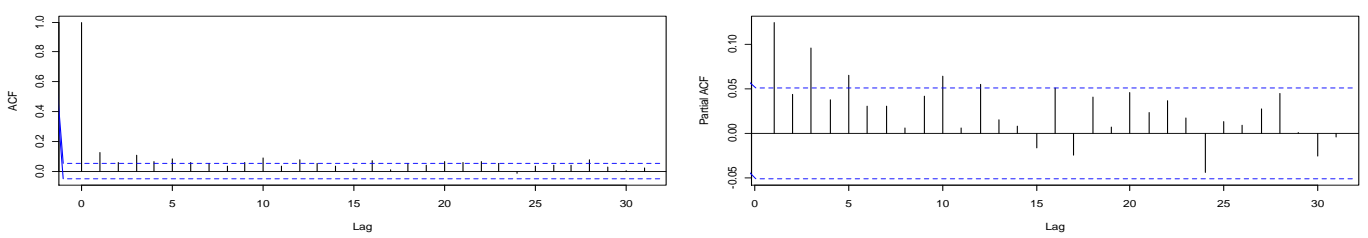

Figure 3. Quantile residuals graphs, LANNE model.

Let's try now to estimate the our time series through the other MEM formulation, the DLG one. This specification is a mixture of two Gamma MEMs with different coefficients for the conditional expectation. The equation for the conditional mean will be:

$$
\mu_{t}=\omega+\alpha_{1} \cdot y_{t-1}+\alpha_{2} \cdot y_{t-2}+\beta_{1} \mu_{t-1}
$$

We use the method of Maximum Likelihood (ML) to estimate the parameters of interest.

\begin{tabular}{|l|l|l|}
\hline Parametro & Stima & p-value \\
\hline$\lambda_{1}$ & 6.723 & $\sim 0$ \\
\hline$\lambda_{2}$ & 16.016 & $\sim 0$ \\
\hline$\omega$ & 0.099 & $\sim 0$ \\
\hline$\alpha_{1}$ & 0.374 & $\sim 0$ \\
\hline$\alpha_{2}$ & 0.081 & 0.805 \\
\hline$\beta_{1}$ & 0.631 & $\sim 0$ \\
\hline$\delta_{0}$ & 0.905 & $\sim 0$ \\
\hline$\delta_{1}$ & 0.983 & $\sim 0$ \\
\hline$\delta_{2}$ & 17.106 & $\sim 0$ \\
\hline$\gamma_{1}$ & 0.419 & $\sim 0$ \\
\hline
\end{tabular}

Table 2. Parameters test, DLG model. 
As we can see from the table, we refuse the $\mathrm{H} 0$ hypothesis in all cases, except for $\alpha_{2}$. The $\mathrm{p}$-value in this case is greater than $5 \%$, so we accept the null hypothesis that the coefficient is statistically equal to zero. We use even in this case the quantile residuals method. We check if residuals are independent and approximate the standard normal distribution. If this is true, the model is correctly specified and the parameters are consistently estimated. If not, quantile residuals are expected to exhibit different characteristics, i.e. dependence, autocorrelation or different distribution.
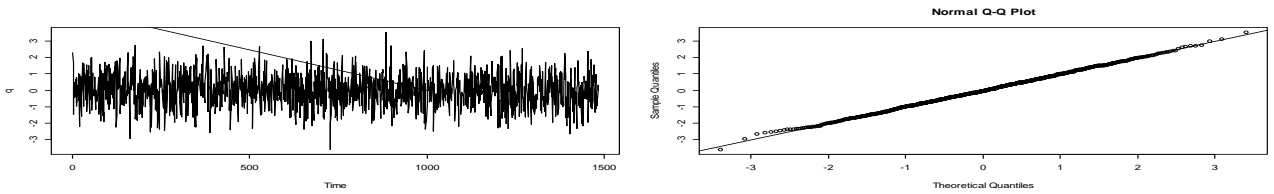

Series q

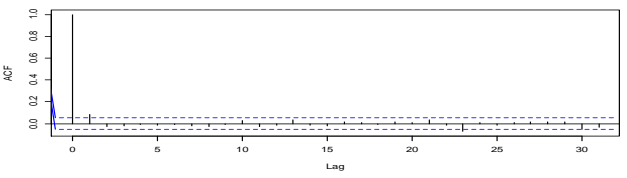

Series a

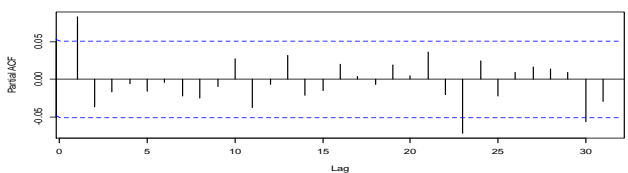

Series $\mathbf{q}^{\wedge 2}$
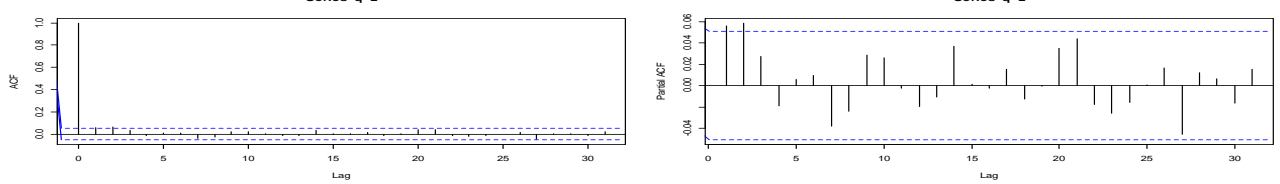

Figure 4. Quantile residuals graphs, DLG model.

We have represented in figure 4 the quantile residual graph, the quantile-quantile graph (if the distribution approximates the normal standard one), the global autocorrelation (ACF) and the partial autocorrelation (PACF) of the quantile residuals and of the squared quantile residuals for the estimated model. We observe that the points line up perfectly to the normal distribution. The global and partial autocorrelations are, in most cases, within the confidence bands. We conclude that the model is correctly specified and the estimates are consistent.

Now we compare our models using well known selection criteria as AIC (Asymptotic Information Criterion; Akaike, 1974) and BIC (Bayesian Information Criterion; Schwarz, 1978). The first criterion, AIC, is defined as:

$A I C(k)=2 \cdot k-2 \cdot l_{T}$,

where $l_{T}$ is the maximized value of the likelihood function for the estimated model, $k$ is the number of parameters in the statistical model and $\mathrm{T}$ is the number of data points. $\mathrm{BIC}$ is defined as:

$B I C(k)=k \cdot \log (T)-2 \cdot l_{T}$.

We have presented in the table below the maximized value of the likelihood function, AIC and BIC for the estimated models.

\begin{tabular}{|l|l|l|l|}
\hline MEM Model & It & AIC & BIC \\
\hline LANNE & $-109,217$ & 236,434 & 281,704 \\
\hline DLG & $-71,638$ & 163,276 & 213,576 \\
\hline
\end{tabular}

Table 3. Maximized value of the likelihood function, AIC and BIC criteria.

Lower values of AIC and BIC, as well as higher values of the maximized value of the likelihood function correspond to the most fitting model and vice versa. In this case, we can conclude that DLG model fits better the corresponding data sample. 


\section{Concluding remarks}

We consider in this study two possible innovative equations for the realized volatility of financial returns, part of the multiplicative error models family, called LANNE and DLG respectively. We estimate and compare these models using some applications on 'STOXX Balkan 50 Equal Weight' Index, for the period from 1 May 2009 to 30 January 2015. The error term of LANNE equation follows a gamma distribution with constant parameters, whereas the error term of DLG equation is distributed according to a mixture of Gamma with variable weights.

We compute the quantile residuals in R 3.1.3 software, in order to measure how well the specific MEM models fit the corresponding data sample. We observe positive signals of independence and a satisfactory approximation of residuals to the standard normal distribution. This implies that the equations fit fairly well the volatility of 'STOXX Balkan 50 Equal Weight' Index, and the corresponding coefficients are consistently estimated. We also calculate the maximized value of the likelihood function, and the AIC and BIC criteria for each model, concluding that DLG model fits better the data. This study can be enriched with supplemental analysis regarding other possible approaches of volatility estimation and other possible variables. Furthermore, we can test and compare these methods through the quantile residuals analysis, as well as through forecasts.

\section{References}

[1] Ahoniemi, K., \& Lanne, M. (2013). Overnight Stock Returns and Realized Volatility. International Journal of Forecasting $29,4,592-604$.

[2] Akaike, H. (1974). A new look at the statistical model identification. IEEE Transactions on Automatic Control 19 (6), $716-$ 723.

[3] Alessi, L., Barigozzi, M., \& Capasso, M. (2009). Estimation and forecasting in large datasets with conditionally heteroskedastic dynamic common factors. Technical Report 09-1115, European Central Bank.

[4] Andersen, T. G., Bollerslev, T., \& Diebold, F. X. (2007). Roughing it up: Including jump components in the measurement, modeling and forecasting of return volatility. Review of Economics and Statistics, 89, 701-720.

[5] Bauwens, L., Hafner, C.M., \& Laurent, S. (2012). Volatility models. Handbook of Volatility Models and their Applications, 1-45. Hoboken, New Jersey: J. Wiley \& Sons.

[6] Bollerslev, T. (1986). Generalized autoregressive conditional heteroskedasticity. Journal of Econometrics 31 (3), 307327.

[7] Bordignon, S., \& Raggi, D. (2012). Long memory and nonlinearities in realized volatility: a Markov switching approach. Computational Statistics and Data Analysis, 56, 3730-3742.

[8] Brownlees, C. T., \& Gallo, G. M. (2006). Financial econometric analysis at ultra-high frequency: Data handling concerns. Computational Statistics and Data Analysis, 51, 2232-2245.

[9] Brownlees, C. T. \& Gallo, G. M. (2010). Comparison of volatility measures: A risk management perspective. Journal of Financial Econometrics, 8, 29-56.

[10] Chiriac, R., \& Voev, V. (2011). Modelling and forecasting multivariate realized volatility. Journal of Applied Econometrics, 26, 922-947.

[11] Brownlees, C.T., Cipollini, F., \& Gallo, G.M. (2011). Multiplicative error models. Econometrics Working Papers Archive 2011_03, Universita' degli Studi di Firenze, Dipartimento di Statistica, Informatica, Applicazioni G. Parenti.

[12] Colacito, R., Engle, R. F., \& Ghysels, E. (2011). A component model for dynamic correlations. Journal of Econometrics, 164, 45-59.

[13] Corsi, F. (2009). A simple approximate long-memory model of realized volatility. Journal of Financial Econometrics 7 , 174-196.

[14] Cox, D. R., Snell, E. J. (1968). A general definition of residuals. Journal of the Royal Statistical Society B, London, v. 30, n. 2, 248-254. 
[15] Crato, N., \& de Lima, P.J.F. (1994). Long-range dependence in the conditional variance of stock returns. Economics Letters, Elsevier, vol. 45(3), 281-285.

[16] De Luca G., Gallo G.M. (2007). Time-varying mixture MEM for realized volatility. S.Co 2007, Book of Short Papers.

[17] Ding, Z., Granger, C. W. J., \& Engle, R. F. (1993). A long memory property of stock market returns and a new model. Journal of Empirical Finance 1, 83-106.

[18] Dunn, P.K., \& Smyth, G.K. (1996). Randomized quantile residuals. Journal of Computational and Graphical Statistics, $5(3), 236-244$.

[19] Engle, R. F., \& Lee, G. J. (1999). A permanent and transitory component model of stock return volatility. Cointegration, causality, and forecasting: A Festschrift in Honor of Clive W. J. Granger, 475-497. Oxford University Press, Oxford.

[20] Engle, R.F. (2002). New frontiers for ARCH models. Journal of Applied Econometrics, 17, 425-446.

[21] Engle, R.F., Gallo, G.M. (2006). A multiple indicators model for volatility using intra-daily data. Journal of econometrics, 131, 3-27.

[22] Engle, R.F., Russell, J.R. (1998). Autoregressive conditional duration: a new model for irregularly spaced transaction data. Econometrica, 66, 1127-1162.

[23] Cipollini, F., Engle, R.F., \& Gallo, G.M. (2006). Vector multiplicative error models: representation and inference. NBER Technical Working Papers 0331, National Bureau of Economic Research, Inc.

[24] Cipollini, F., Engle, R.F., \& Gallo, G.M. (2013). Semiparametric vector MEM. Journal of Applied Econometrics, 28(7), 1067-1086.

[25] Gallo, G., \& Otranto, E. (2014). Forecasting Realized Volatility with Changes of Regimes. Econometrics Working Papers Archive 2014_03. Universita' degli Studi di Firenze, Dipartimento di Statistica, Informatica, Applicazioni "G. Parenti".

[26] Granger, C. W. J., \& Joyeux, R. (1980). An introduction to long-memory time series models and fractional differencing. Journal of Time Series Analysis 1, 15-30.

[27] Hansen, P. R., Huang, Z., \& Shek, H. H. (2012). Realized GARCH: A joint model of returns and realized measures of volatility. Journal of Applied Econometrics, 27, 877-906.

[28] Hosking, J.R.M. (1981). Fractional differencing. Biometrika 68(1), 165-176.

[29] Lanne, M. (2006). A mixture multiplicative error model for realized volatility. Journal of Financial Econometrics, 4, 594616.

[30] Barigozzi, M., Brownlees, C.T., Gallo, G.M., \& Veredas, D. (2014). Disentangling systematic and idiosyncratic dynamics in panels of volatility measures. Econometrics Working Papers Archive 2014_02, Universita' degli Studi di Firenze, Dipartimento di Statistica, Informatica, Applicazioni G. Parenti.

[31] Patton, A. (2010). Volatility forecast comparison using imperfect volatility proxies. Journal of Econometrics, 160, 246256.

[32] Rosenblatt, M. (1952). Remarks on a multivariate transformation. Annals of Mathematical Statistics, 23, 470-472.

[33] Schwarz, G.E. (1978). Estimating the dimension of a model. Annals of Statistics 6 (2), 461-464. 
